\title{
Development of the Ubiquitous Spaced Retrieval-Based Memory Advancement and Rehabilitation Training Program
}

\author{
Ji Won Han', Kyusoo Oh¹, Sooyoung Yoo², Eunhye Kim², Ki-Hwan Ahn³, \\ Yeon-Joo Son ${ }^{3}$, Tae Hui Kim¹, Yeon Kyung Chi', and Ki Woong Kim ${ }^{1,4,5} \bowtie$ \\ ${ }^{1}$ Department of Neuropsychiatry, Seoul National University Bundang Hospital, Seongnam, Republic of Korea \\ ${ }^{2}$ Center for Medical Informatics, Seoul National University Bundang Hospital, Seongnam, Republic of Korea \\ ${ }^{3}$ Technology Development Office, Advanced Institute of Technology, KT R\&D Center, Seoul, Republic of Korea \\ ${ }^{4}$ Department of Psychiatry and Behavioral Sciences, Seoul National University College of Medicine, Seoul, Republic of Korea \\ ${ }^{5}$ Department of Brain and Cognitive Science, Seoul National University College of Medicine Sciences, Seoul, Republic of Korea
}

Objective The Ubiquitous Spaced Retrieval-based Memory Advancement and Rehabilitation Training (USMART) program was developed by transforming the spaced retrieval-based memory training which consisted of 24 face-to-face sessions into a self-administered program with an iPAD app. The objective of this study was to evaluate the feasibility and efficacy of USMART in elderly subjects with mild cognitive impairment (MCI).

Methods Feasibility was evaluated by checking the satisfaction of the participants with a 5-point Likert scale. The efficacy of the program on cognitive functions was evaluated by the Korean version of the Consortium to Establish a Registry for Alzheimer's Disease Neuropsychological Assessment Battery before and after USMART.

Results Among the 10 participants, 7 completed both pre- and post-USMART assessments. The overall satisfaction score was $8.0 \pm 1.0$ out of 10. The mean Word List Memory Test (WLMT) scores significantly increased after USMART training after adjusting for age, educational levels, baseline Mini-Mental Status Examination scores, and the number of training sessions (pre-USMART, 16.0 \pm 4.1 ; postUSMART, $17.9 \pm 4.5 ; \mathrm{p}=0.014, \mathrm{RM}$-ANOVA). The magnitude of the improvements in the WLMT scores significantly correlated with the number of training sessions during 4 weeks $(r=0.793 ; \mathrm{p}=0.033)$.

Conclusion USMART was effective in improving memory and was well tolerated by most participants with MCI, suggesting that it may be a convenient and cost-effective alternative for the cognitive rehabilitation of elderly subjects with cognitive impairments. Further studies with large numbers of participants are necessary to examine the relationship between the number of training sessions and the improvements in memory function.

Psychiatry Investig 2014;11:52-58

Key Words Mild cognitive impairment, Cognitive therapy, Rehabilitation, Memory, Computer user training, Dementia.

\section{INTRODUCTION}

Alzheimer's disease $(\mathrm{AD})$ is one of the most distressing and burdensome mental health problems in the aged population, and the incidence of dementia is continuously increasing. Although pharmacological agents, such as acetylcholinesterase inhibitors and N-methyl-D-aspartate receptor antagonists,

Received: February 4, 2013 Revised: April 9, 2013

Accepted: April 9, 2013 Available online: January 21, 2014

$\triangle$ Correspondence: Ki Woong Kim, MD, PhD

Department of Neuropsychiatry, Seoul National University Bundang Hospital, 166 Gumi-ro, Bundang-gu, Seongnam 463-707, Republic of Korea Tel: +82-31-787-7432, Fax: +82-31-787-4058, E-mail: kwkimmd@snu.ac.kr

(a) This is an Open Access article distributed under the terms of the Creative Commons Attribution Non-Commercial License (http://creativecommons.org/licenses/by$\mathrm{nc} / 3.0$ ) which permits unrestricted non-commercial use, distribution, and reproduction in any medium, provided the original work is properly cited. have demonstrated efficacy in the temporal symptomatic control of the cognitive decline and daily function of patients with $\mathrm{AD}$, their efficacy has not been sufficient to restore premorbid function or maintain functional levels in the later stages of patients with AD. ${ }^{1}$ Furthermore, no effective medication has been found for patients with mild cognitive impairment (MCI), which is a high-risk condition for AD.

Therefore, a multifactorial therapeutic approach that includes both pharmacological and non-pharmacological interventions is being increasingly advocated in order to optimize the cognition, affect, and global functioning of patients with $\mathrm{MCI}$ or $\mathrm{AD}{ }^{2,3}$ Because memory impairment is the earliest core symptom of $\mathrm{AD}$, memory rehabilitation, such as the spaced retrieval training (SRT), is a critical part of the non-pharmacological treatments for AD. ${ }^{1,4}$ SRT is a method of learning and 
retaining target information by recalling that information over increasingly longer intervals. ${ }^{5}$ It was originally developed for improving memory in cognitively intact persons and was based on the expanding rehearsal technique., ${ }^{6,7}$ The modification was designed to reduce the learner's effort during training, reduce the number of errors by matching the difficulty of the task to the patient's ongoing performance (shaping paradigm), and make the training sessions social and enjoyable. ${ }^{7}$ SRT has been reported to improve learning and target information retention and to ameliorate behavioral problems in various types of dementia, including $\mathrm{AD} .^{7-12}$

In our previous work, we developed a Spaced Retrievalbased Memory Advancement and Rehabilitation Training (SMART) program, which consisted of 24 face-to-face sessions. ${ }^{13}$ SMART was effective in improving the memory retention spans of patients with very mild to mild AD. Furthermore, the expanded retention span induced by SMART was maintained for different sets of target information, indicating that the efficacy of SMART can be generalized.

However, SMART has several limitations in its current form that prevent it from being provided to as many individuals who may benefit from it as possible. First, more than an hour of face-to-face contact with a trained therapist is required for each session, and, thus, it has high costs. Furthermore, trained therapists are not available in many areas. Second, it is not easy to maintain compliance because both patients and caregivers need to regularly allow for about 3 hours per visit and 3 visits a week. Third, it is difficult to increase the intensity of the training, such as the length of the retention span and the number of training sessions per week, due to the previously described limitations. In order to overcome these limitations of SMART, we developed a Ubiquitous SMART (U-SMART) program by transforming the current SMART program into a self-administered program with an $\mathrm{PAD}$ app, and we examined its feasibility and efficacy in elderly individuals with MCI.

\section{METHODS}

\section{Subjects}

Ten elderly individuals with MCI participated in the present study; 4 were enrolled from the participants of the Korean Longitudinal Study on Cognitive Aging and Dementia (KLOSCAD), and 6 were enrolled from the visitors to the Dementia Clinic of the Seoul National University Bundang Hospital. The KLOSCAD is a population-based prospective elderly cohort study on cognitive aging and dementia that was launched in 2009.

Each subject was evaluated with the Korean version of the Consortium to Establish a Registry for Alzheimer's Disease $(\mathrm{CERAD}-\mathrm{K})^{14}$ by a research geropsychiatrist with expertise in dementia research. MCI was diagnosed according to the Revised Diagnostic Criteria for MCI proposed by the International Working Group on MCI. ${ }^{15}$ The subjects who had Axis I disorders that are listed in the DSM-IV ${ }^{16}$ or serious medical or neurological disorders that could affect their cognitive function were excluded.

This study's protocol was approved by the Institutional Review Board of Seoul National University Bundang Hospital (SNUBH). All subjects were fully informed of the study protocol. All subjects provided written informed consents that were signed by the subjects.

\section{Development of USMART}

USMART was based on a client-server model in which all data were stored in a central server, while the iPAD mobile application was designed and implemented in order to provide patients with a self-administered SRT program. A web portal was developed to provide service providers, such as therapists, with accessibility and management capabilities of the data (Figure 1). The client program and server system communicated over the network with a Hypertext Transfer Protocol Secure (HTTPS) in order to ensure secure communication.

As shown in Figure 1, the central server consisted of the following different layers of servers in order to support the efficient development of the mobile and web applications: the open application programming interface (API), which acted as a gateway between the mobile application and central storage; the controller layer, which provided a web service for providers; the data access layer, which provided queries to access the underlying database; and the database server in the persistence layer. All server programs were implemented on a Java platform.

The iPAD mobile application, which was a SRT program for patients, was designed to be elderly-friendly and easy to use by using high-chroma colors, minimizing user options, providing step-by-step voice-guided directions, and providing various types of input modes, such as voice recognition, keyboard typing, and writing recognition. With the application, patients are allowed to have a training session whenever they want, regardless of their location and time. Their training records are then automatically sent to and stored in the central database through the open API. The current mobile application was tuned to operate with a set of 88 words. However, it also provided a functionality that allowed patients to train with their own userdefined words. One hundred and twenty functional games were embedded and provided to patients as part of the interretrieval activities.

The development of a web portal for providers enabled providers to register a new patient, access the patient's training history, check the patient's progress, and evaluate the training results. 


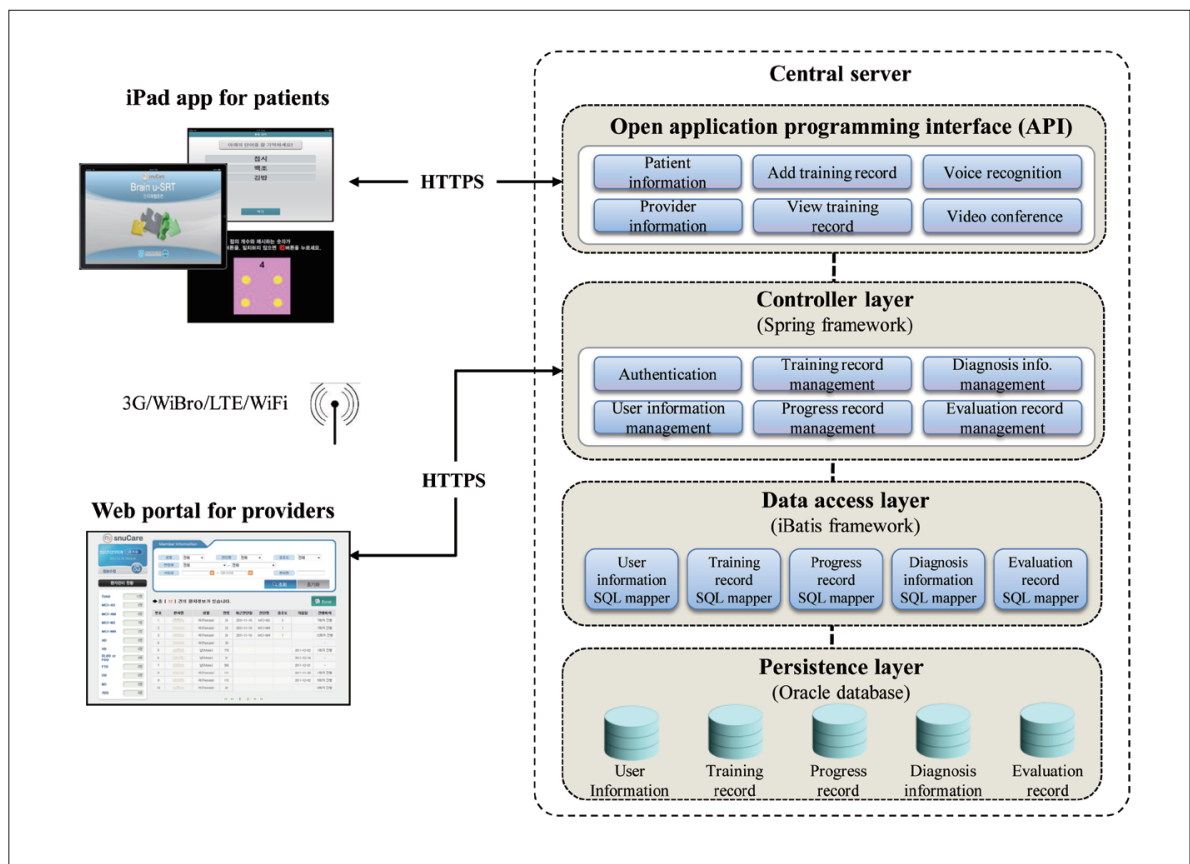

Figure 1. System architecture of the Ubiquitous Spaced Retrieval-based Memory Advancement and Rehabilitation Training (USMART).

\section{Administration of USMART}

Two occupational therapists trained each participant on how to use the USMART application and IPAD. Then, each participant was asked to use the USMART 3 or more times per week for 4 weeks at home. During the 4 weeks, the participants were allowed to ask the 2 research occupational therapists for directions of the USMART anytime by phone or at a hospital visit.

In each USMART session, the subject was asked to remember a given set of words that appeared in the screen and then recall them immediately. The subject could recall by voice recognition or direct keyboard typing. If the subject succeeded in recalling the words, he/she was asked to recall them repeatedly over systematically expanded intervals $(0.75,1.5,3,6,12$, and $24 \mathrm{~min}){ }^{13}$ Following a recall failure, the interval was reduced to that of the previous trial. The set of words consisted of 1 to 5 unrelated high-imagery words (e.g., bus, grape, duck, scissors, etc.). All targets were disyllabic words in the Korean language, and different words were used in all sessions. ${ }^{13}$ If the subject succeeded in recalling the given set of words for $24 \min$ in 3 consecutive sessions, the number of words in the set was sequentially increased in the subsequent session by an automated algorithm in the USMART. The starting number of words in the first session was 1 . During each interval, the subjects were asked to perform inter-retrieval activities in order to prevent them from rehearsing the given set of words. The inter-retrieval activities consisted of a quiz that could improve attention/concentration, visual memory, orientation, and categorization. All procedures during the USMART training were guided by automatic verbal messages in the
USMART apparatus (i.e., the iPAD).

\section{Assessments of feasibility and efficacy}

We evaluated the feasibility of USMART by assessing the satisfaction and compliance of the participants. We measured the level of satisfaction with USMART with a 17-item satisfaction questionnaire (4 items on the content of the program, 2 items on accuracy, 2 items on the layout of the program, 2 items on the ease of use, 2 items on timeliness, 3 items on the program speed, and 2 items on overall satisfaction). Each item was scored with a 5-point Likert scale from 1 (not at all) to 5 (very much).

We evaluated compliance by assessing the number of selftraining sessions that were performed for 4 weeks.

In order to evaluate the efficacy of USMART on cognitive functions, we administered the Korean version of the CERAD Neuropsychological Assessment Battery (CERAD-K-N), the Korean version of the Frontal Assessment Battery, ${ }^{17}$ and the Digit Span Test ${ }^{18}$ before and after subjects performed the USMART program. The CERAD-K-N consists of 9 neuropsychological tests, including the Categorical Fluency test, the Modified Boston Naming Test, the Mini-Mental State Examination (MMSE), the Word List Memory Test (WLMT), the Constructional Praxis Test, the Word List Recall Test, the Word List Recognition Test, the Constructional Recall Test, and the Trail Making Test A. These pre- and post-treatment assessments were performed within 2 weeks before or after the performance of the 4-weeks' USMART program. 


\section{Statistical analysis}

The Mann-Whitney U-test was used to compare the age, educational levels, and baseline MMSE scores between the subjects who completed the U-SMART program and those who dropped out.

Repeated measures analysis of variance (RM-ANOVA) was used to compare the pre- and post-USMART neuropsychological performances after adjusting for age, educational levels, pre-USMART MMSE scores, and the number of training sessions that were performed for 4 weeks. For any of the neuropsychological tests that showed improvement by the training, a correlation analysis using Spearman's rank correlation coefficient was performed to examine the factors that determined the degree of improvement. For our statistical evaluation of the main effects, $P$ values less than 0.05 were considered statistically significant. All statistical analyses were performed with the PASW Statistics 18, Release Version 18.0.0 (Ó SPSS, Inc., Chicago, IL, USA).

\section{RESULTS}

The demographic characteristics of the 10 participants (1 man, 9 women) are summarized in Table 1. Among them, 7 women ( 4 amnestic MCI single-domain type, 2 amnestic MCI multiple-domain type, 1 non-amnestic MCI single-domain type) completed both the pre- and post-USMART assessments. The number of training sessions that were performed by these 7 participants for 4 weeks varied widely from 1 to 30 . Among them, 1 subject (ID 7) had been taking donepezil (10 $\mathrm{mg}$ ) for 6 months at the beginning of this study. Three subjects dropped out for the following reasons: 1 subject (ID 8) had difficulty learning how to use the iPAD, 1 (ID 9) felt the interretrieval activities were childish and 1 (ID 10) developed an acute physical illness. However, their age ( $\mathrm{p}=0.085$, Mann-
Whitney U-test), education ( $\mathrm{p}=0.816$, Mann-Whitney U-test), and baseline MMSE scores ( $\mathrm{p}=0.728$, Mann-Whitney U-test) were comparable to those of the 7 subjects who completed the assessments.

The overall satisfaction score was $8.0 \pm 1.0$ out of 10 points. All participants scored 4 or 5 in the overall satisfaction items and answered that they were willing to purchase the USMART program. Five responders (71.4\%) answered that the USMART was helpful in improving their memory. The points for 15 out of the 17 items were 3 or more. Mean scores of the content, accuracy, layout, ease of use, timeliness, and program speed were $12.7 \pm 3.1$ out of 20 points, $7.4 \pm 1.5$ out of 10 points, $7.3 \pm 1.5$ out of 10 points, $7.1 \pm 1.7$ out of 10 points, $6.6 \pm 2.0$ out of 10 points, and $9.4 \pm 2.9$ out of 15 points, respectively.

As shown in Table 2, the mean WLMT score significantly increased after the USMART training (pre-USMART, $16.0 \pm$ 4.1; post-USMART, 17.9 \pm 4.5 ). After adjusting for age, educational level, baseline MMSE score, and the number of training sessions, this change was statistically significant $(\mathrm{F}(1,2)$ $=70.327, \mathrm{p}=0.014$ ). Figure 2 shows the changes in the WLMT scores after USMART in each participant. Furthermore, the magnitudes of the improvements in the WLMT scores significantly correlated with the number of training sessions performed during the 4 weeks ( $r=0.793, \mathrm{p}=0.033$ ) (Figure 3).

\section{DISCUSSION}

We found that performing the USMART program for 4 weeks was applicable to a considerable proportion of the elderly patients with MCI, and it was helpful in improving word list learning in proportion to the number of training sessions.

We were concerned whether elderly people with cognitive impairments were able to use the USMART program by themselves. However, most participants seemed to adapt readily to

Table 1. The characteristics of the participants

\begin{tabular}{ccccccc}
\hline ID & Age $(\mathrm{y})$ & Gender & Education $(\mathrm{y})$ & Diagnosis & CDR & Session* \\
\hline 1 & 61 & Female & 9 & MCI-AM & 0.5 & $30(44)$ \\
2 & 67 & Female & 6 & MCI-AS & 0.5 & $7(14)$ \\
3 & 60 & Female & 6 & MCI-AS & 0.5 & $13(19)$ \\
4 & 74 & Female & 6 & MCI-AS & 0.5 & $21(28)$ \\
5 & 73 & Female & 16 & MCI-NS & 0.5 & $12(12)$ \\
6 & 73 & Female & 12 & MCI-AS & 0.5 & $7(12)$ \\
7 & 80 & Female & 9 & MCI-AM & 0.5 & $1(3)$ \\
8 & 79 & Female & 5 & MCI-AM & 0 & 0 \\
9 & 79 & Female & 10 & MCI-AM & 0.5 & 0 \\
10 & 75 & Male & 16 & MCI-AS & 0 & 0 \\
\hline
\end{tabular}

*total number of training sessions during 4 weeks. MCI: mild cognitive impairment, AM: amnestic multiple-domain type, AS: amnestic single-domain type, NS: non-amnestic single-domain type, CDR: Clinical Dementia Rating 
Table 2. The effect of Ubiquitous Spaced Retrieval-based Memory Advancement and Rehabilitation Training (USMART) on the cognitive functions of elderly individuals with mild cognitive impairment (MCI)

\begin{tabular}{|c|c|c|c|c|}
\hline & Pre-USMART & Post-USMART & $\mathrm{p}^{*}$ & $\mathrm{p}^{\ddagger}$ \\
\hline Mini-Mental State Examination & $26.7 \pm 1.9$ & $25.2 \pm 1.8$ & 0.068 & \\
\hline Categorical Fluency Test & $12.0 \pm 3.2$ & $11.3 \pm 2.4$ & 0.343 & 0.401 \\
\hline Boston Naming Test & $11.4 \pm 2.3$ & $11.9 \pm 0.9$ & 0.671 & 0.756 \\
\hline Word List Memory Test & $16.0 \pm 4.1$ & $17.9 \pm 4.5$ & 0.246 & 0.014 \\
\hline Word List Recall Test & $4.4 \pm 2.2$ & $5.4 \pm 3.6$ & 0.345 & 0.279 \\
\hline Word List Recognition Test & $8.3 \pm 1.8$ & $8.6 \pm 1.9$ & 0.891 & 0.847 \\
\hline Construction Praxis Test & $10.1 \pm 1.5$ & $10.4 \pm 0.8$ & 0.414 & 0.191 \\
\hline Construction Recall Test & $4.9 \pm 3.0$ & $5.6 \pm 3.0$ & 0.496 & 0.598 \\
\hline Trail Making Test-A & $51.0 \pm 15.4$ & $52.6 \pm 13.3$ & 0.498 & 0.836 \\
\hline Trail Making Test-B & $204.1 \pm 76.0$ & $188.0 \pm 74.7$ & 0.128 & 0.063 \\
\hline
\end{tabular}

${ }^{*}$ Wilcoxon signed-rank test, ${ }^{\ddagger}$ repeated Measured Analysis of Variance (RM-ANOVA), adjusted for age, educational level, MMSE score before the USMART, and the number of training sessions for 4 weeks

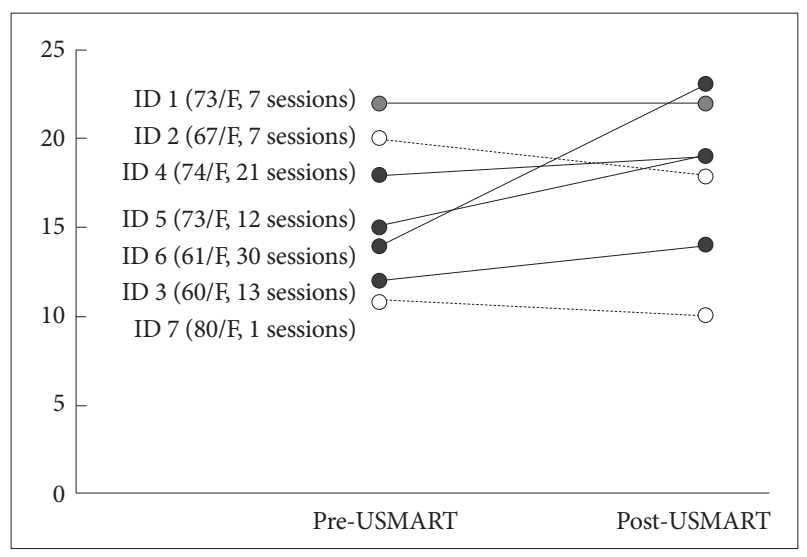

Figure 2. The changes in the Word List Memory Test scores after USMART in each participant. MCl-AM: mild cognitive impairmentamnestic multiple-domain type, AS: amnestic single-domain type, NS: non-amnestic single-domain type.

the USMART program after several introductions and did not need to have a caregiver or therapist present during training, indicating that the USMART program may not be overwhelming or emotionally taxing to elderly patients with MCI. However, 1 participant dropped out because he failed to learn how to use the iPAD, and 5 of the 7 participants who completed the current study needed 2 or more teaching sessions to learn how to use the iPAD properly. In addition, the participants who had difficulty learning how to use the iPAD had a lower number of training sessions. If smart devices like the iPAD will become more popular and familiar to elderly individuals in the future, the USMART program will become more widely and easily applicable to elderly patients with cognitive impairments. The 7 participants showed high levels of overall satisfaction. However, their satisfaction with each aspect of the program and its timeliness and speed in particular was somewhat lower than their overall satisfaction. In addition, some participants felt that the inter-retrieval activities were too easy and childish.

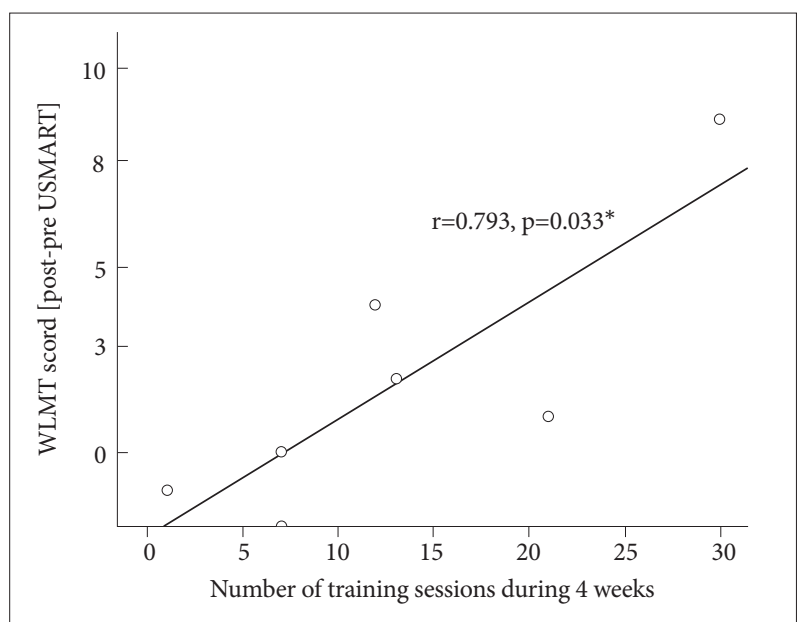

Figure 3. Correlation between the number of training sessions and the magnitude of the improvements in the Word List Memory Test (WLMT) scores. *Spearman's rank correlation coefficient.

The inter-retrieval activities were tailored to each subject in order to enhance the compliance with the USMART program.

We found that USMART dose-dependently improved the memory of elderly individuals with MCI. These data add to the growing empirical evidence on the benefits of spaced retrieval on the memory function of patients with MCIs. ${ }^{19}$ In this study, the efficacy of the USMART program was confined to memory aspects that were measured by the WLMT. Our observation was consistent with earlier studies that reported that objective measures of memory reveal statistically significant improvements at the end of training when compared to objective measures of cognition other than memory. ${ }^{2}$ The effectiveness of spaced retrieval as a method of facilitating memory function has been variously attributed to the effects of repetition priming, operant conditioning, classical conditioning, and errorless learning, ${ }^{12,20,21}$ but there is a general consensus that at least some of the effects of spaced retrieval are attributable to 
its reliance on automatic processing. ${ }^{20-22}$ Different cognitive processes are subjected to different rates and degrees of decline in both patients with dementia and MCI; controlled cognitive processing is more substantially affected than automatic processing. ${ }^{23}$ The USMART program may be helpful for improving the storage of target information in the everyday lives of patients with MCI.

Furthermore, the magnitude of memory improvement was correlated with the number of training sessions in the present study, which further supported the findings that the USMART program may be effective in improving memory. Some studies have suggested that participants become more proficient at the SRT task with an increasing number of sessions, which is reflected in the longer retention intervals in the later sessions compared to the early sessions and in the number of failures that decreased for each participant across the sessions. ${ }^{24-26}$ These explanations provide additional evidence that, over time, participants benefit from prior training sessions. However, the direct effect of the increased training sessions on cognitive improvement has not been fully investigated yet. One previous study that included 60 training session $\mathrm{s}^{27}$ did not show any superiority compared to the other studies involving an inferior number of sessions, and others have suggested that those programs that apply fewer sessions (between 6 and 20) seem to be more cost-effective for future research and clinical purposes. ${ }^{2}$ The number of training sessions for the 4 weeks in our study had a mean value of 13 (s.d., 9.7). Therefore, the duration of our study might not be categorized as long term. Further studies that include a large number of participants are necessary to examine the relationship between the number of training sessions and the improvements in memory function.

According to the current study, the memory-enhancing effects of the USMART program might be generalized because the performance of the word list learning test, which employed a set of words that were totally different from those employed in the USMART program, was significantly improved after the USMART program. In our previous study on the SMART program, however, SMART was effective in expanding the retention span of words that were very different from the words that were employed in the SMART training but not in improving the performance of the word list learning test. ${ }^{13}$ This discrepancy between the effects of USMART and SMART on the word list learning test may be partly attributed to differences in the severity of the cognitive impairments in the subjects in the 2 studies. In the previous study on SMART, all subjects were $\mathrm{AD}$ patients, whereas all were MCI patients in the present study. Because more cognitive effort and explicit memory may be required for conventional neuropsychological tests like the word list learning test compared to SMART or USMART, ${ }^{6,12}$ the effect size of SMART or USMART on the performance of the WLMT may be smaller in AD patients who have more severe impairments in explicit memory than in individuals with MCI.

Although the current study showed that USMART might be feasible and useful in elderly individuals with MCI, several limitations should be noted. First, the sample size was small, the treatment period was short, and the study design was an open-label single arm. Second, the type of MCI was not uniform. The feasibility and efficacy of the USMART may be different depending on the type of MCI, i.e., amnestic versus non-amnestic. Third, long-term efficacy was not evaluated in the present study. Because MCI is a degenerative condition in at least a certain proportion of individuals, ${ }^{28-30}$ it is crucial to assess the longitudinal course of the efficacy data. A delay of the conversion toward dementia could eventually be considered the ultimate efficacy outcome. Fourth, medication effects were not totally excluded in this study. One subject had been taking donepezil, and 1 subject had been taking escitalopram. However, the changes in the WLMT scores after USMART did not differ in the subjects taking these drugs. The WLMT score of the subject who was taking donepezil decreased 1 point and that of the subject who was taking escitalopram increased 1 point after USMART.

In conclusion, USMART was effective in improving memory, and it was well tolerated by most participants with MCI, suggesting that it may be a convenient and cost-effective alternative for cognitive rehabilitation in elderly patients with cognitive impairments. Information technology can play a key role in making conventional cognitive training more accessible and convenient to elderly patients with cognitive impairments.

\section{Acknowledgments}

This work was supported by the KT-SNUBH Collaborative Research Fund and a grant from the Korean Health Technology R\&D Project, Ministry for Health, Welfare, \& Family Affairs, Republic of Korea (Grant No. A092077). Kim KW serves as an editorial board member of the Psychiatry Investigation, receives research support from Esai Korea, Wyeth Pharmaceuticals, Eli Lilly, H. Lundbeck A/S, and receives research support from the Ministry of Health and Welfare, Korea.; Han JW, Oh KS, Yoo SY, Kim $\mathrm{EH}, \mathrm{Ahn} \mathrm{KH}$, Son YJ, Kim TH and Chi YK had no disclosures.

\section{REFERENCES}

1. O'Hara R, Mumenthaler MS, Yesavage JA. Update on Alzheimer's disease: recent findings and treatments. West J Med 2000;172:115-120.

2. Jean L, Bergeron ME, Thivierge S, Simard M. Cognitive intervention programs for individuals with mild cognitive impairment: systematic review of the literature. Am J Geriatr Psychiatry 2010;18:281-296.

3. Yesavage JA, Westphal J, Rush L. Senile dementia: combined pharmacologic and psychologic treatment. J Am Geriatr Soc 1981;29:164-171.

4. Choo IH, Lee DY, Lee JH, Kim KW, Jhoo JH, Ju YS, et al. The prevalence of cognitive impairment with no dementia in older people: The Seoul Study. Int J Geriatr Psychiatry 2009;24:306-312.

5. Camp CJ. Facilitation of New Learning in Alzheimer's Disease. New York: Springer; 1989.

6. Camp CJ, Foss JW, O’Hanlon AM, Stevens AB. Memory interventions 
for persons with dementia. Appl Cogn Psychol 1996;10:193-210.

7. Camp CJ, Stevens AB. Spaced-retrieval: a memory intervention for dementia of the Alzheimer's type(DAT). Clinical gerontologist. J Aging Ment Health 1990;10:58-61.

8. Abrahams JP, Camp CJ. Maintenance and generalization of object naming training in anomia associated with degenerative dementia. Clinical Gerontologist: The Journal of Aging and Mental Health 1993; 12:57-72.

9. Bird M, Alexopoulos P, Adamowicz J. Success and failure in five case studies: used of cued recall to ameliorate behaviour problems in senile dementia. Int J Geriatr Psychiatry 1995;10:305-311.

10. Bird M. Clinical use of preserved learning capacity in dementia. Australas J Ageing 1998;17:161-166.

11. Bird M. Behavioural difficulties and cued recall of adaptive behaviour in dementia: Experimental and clinical evidence. Neuropsychol Rehabil 2001;11:357-375.

12. Cherry KE, Simmons-D'Gerolamo SS. Long-term effectiveness of spaced-retrieval memory training for older adults with probable Alzheimer's disease. Exp Aging Res 2005;31:261-289.

13. Lee SB, Park CS, Jeong JW, Choe JY, Hwang YJ, Park CA, et al. Effects of spaced retrieval training (SRT) on cognitive function in Alzheimer's disease (AD) patients. Arch Gerontol Geriatr 2009;49:289-293.

14. Lee JH, Lee KU, Lee DY, Kim KW, Jhoo JH, Kim JH, et al. Development of the Korean version of the Consortium to Establish a Registry for Alzheimer's Disease Assessment Packet (CERAD-K): clinical and neuropsychological assessment batteries. J Gerontol B Psychol Sci Soc Sci 2002;57:P47-P53.

15. Winblad B, Palmer K, Kivipelto M, Jelic V, Fratiglioni L, Wahlund LO, et al. Mild cognitive impairment--beyond controversies, towards a consensus: report of the International Working Group on Mild Cognitive Impairment. J Intern Med 2004;256:240-246.

16. American Psychiatric Association. Diagnostic and statistical manual of mental disorders. 4th edition. Washington, DC: American Psychiatric Association; 1994.

17. Kim TH, Huh Y, Choe JY, Jeong JW, Park JH, Lee SB, et al. Korean version of frontal assessment battery: psychometric properties and normative data. Dement Geriatr Cogn Disord 2010;29:363-370.

18. Wechsler D. Wechsler Memory Scale-Revised. New York: Psychologi- cal Corporation; 1987.

19. Backman L. Memory training and memory improvement in Alzheimer's disease: rules and exceptions. Acta Neurol Scand Suppl 1992;139: 84-89.

20. McKitrick LA, Camp CJ, Black FW. Prospective memory intervention in Alzheimer's disease. J Gerontol 1992;47:p337-P343.

21. Camp CJ. Spaced Retrieval: A Model for Dissemination of a Cognitive Intervention for Persons with Dementia. In: Welsh-Bohmer DKAKA, Editor. Geriatric Neuropsychology: Assessment and Intervention. New York, US: Guilford Publications, 2006;p.275-p.292.

22. Bird M, Kinsella G. Long-term cued recall of tasks in senile dementia. Psychol Aging 1996;11:45-56.

23. Adam S, Van der Linden M, Collette F, Lemauvais L Salmon E. Further exploration of controlled and automatic memory processes in early alzheimer's disease. Neuropsychology 2005;19:420-427.

24. Camp CJ, Burant CJ, Graham GC. The InterpreCare System: overcoming language barriers in long-term care. Gerontologist 1996;36:821-823.

25. Hawley KS, Cherry KE. Spaced-retrieval effects on name-face recognition in older adults with probable Alzheimer's disease. Behav Modif 2004;28:276-296.

26. Ozgis S, Rendell PG, Henry JD. Spaced retrieval significantly improves prospective memory performance of cognitively impaired older adults. Gerontology 2009;55:229-232.

27. Rozzini L, Costardi D, Chilovi BV, Franzoni S, Trabucchi M, Padovani A. Efficacy of cognitive rehabilitation in patients with mild cognitive impairment treated with cholinesterase inhibitors. Int J Geriatr Psychiatry 2007;22:356-360.

28. Lehrner J, Gufler R, Guttmann G, Maly J, Gleiss A, Auff E, et al. Annual conversion to alzheimer disease among patients with memory complaints attending an outpatient memory clinic: the influence of amnestic mild cognitive impairment and the predictive value of neuropsychological testing. Wien Klin Wochenschr 2005;117:629-635.

29. Fischer P, Jungwirth S, Zehetmayer S, Weissgram S, Hoenigschnabl S, Gelpi E, et al. Conversion from subtypes of mild cognitive impairment to Alzheimer dementia. Neurology 2007;68:288-291.

30. Palmer K, Backman L, Winblad B, Fratiglioni L. Mild cognitive impairment in the general population: occurrence and progression to Alzheimer disease. Am J Geriatr Psychiatry 2008;16:603-611. 